\title{
CXCL4 and CXCL10 predict risk of fatal cerebral malaria
}

\author{
Nana O. Wilson ${ }^{\mathrm{a}}$, Vidhan Jain ${ }^{\mathrm{b}}$, Christina E. Roberts ${ }^{\mathrm{c}}$, Naomi Lucchi ${ }^{\mathrm{d}}$, Pradeep K. Joel ${ }^{\mathrm{e}}$, \\ Mrigendra P. Singh ${ }^{\mathrm{b}}$, Avinash C. Nagpal ${ }^{\mathrm{e}}$, Aditya P. Dash ${ }^{\mathrm{f}}$, Venkatachalam Udhayakumar ${ }^{\mathrm{d}}$, \\ Neeru Singh ${ }^{\mathrm{b}, \mathrm{g}}$ and Jonathan K. Stiles ${ }^{\mathrm{a}, *}$ \\ ${ }^{a}$ Morehouse School of Medicine, Department of Microbiology, Biochemistry and Immunology, Atlanta, Georgia, \\ USA \\ ${ }^{\mathrm{b}}$ National Institute of Malaria Research Field Unit (ICMR), Jabalpur, Madhya Pradesh, India \\ ${ }^{\mathrm{c}}$ Spelman College, Biology Department, Atlanta, Georgia, USA \\ ${ }^{\mathrm{d}}$ Malaria Branch, Division of Parasitic Diseases and Malaria, Center for Global Health, Centers for Disease \\ Control and Prevention (CDC), Atlanta, Georgia, USA \\ ${ }^{\mathrm{e}}$ Nethaji Subhash Chandra Bose Medical College Hospital, Jabalpur, Madhya Pradesh, India \\ ${ }^{\mathrm{f}}$ National Institute of Malaria Research (ICMR), New Delhi, India \\ ${ }^{\mathrm{g}}$ Regional Medical Research Center for Tribals (ICMR), Jabalpur, Madhya Pradesh, India
}

\begin{abstract}
Plasmodium falciparum in a subset of patients can lead to a diffuse encephalopathy known as cerebral malaria (CM). Despite treatment, mortality caused by CM can be as high as $30 \%$ while $10 \%$ of survivors of the disease may experience shortand long-term neurological complications. The pathogenesis of CM involves alterations in cytokine and chemokine expression, local inflammation, vascular injury and repair processes. These diverse factors have limited the rate of discovery of prognostic predictors of fatal CM. Identification of reliable early predictors of $\mathrm{CM}$ severity will enable clinicians to adjust this risk with appropriate management of CM. Recent studies revealed that elevated levels of CXCL10 expression in cerebrospinal fluid and peripheral blood plasma independently predicted severe and fatal CM. CXCR3, a promiscuous receptor of CXCL10, plays an important role in pathogenesis of mouse model of CM. In this study the role of corresponding CXCR3 ligands (CXCL11, CXCL10, CXCL9 \& CXCL4) in fatal or severe CM was evaluated by comparing their levels in 16 healthy control (HC), 26 mild malaria (MM), 26 cerebral malaria survivors (CMS) and 12 non-survivors (CMNS) using enzyme linked immunosorbent assay (ELISA). Levels of CXCL4 and CXCL10 were significantly elevated in CMNS patients $(p<0.05)$ when compared with HC, MM and CMS. Elevated plasma levels of CXCL10 and CXCL4 were tightly associated with CM mortality. Receiver Operating Characteristic (ROC) curve analysis revealed that CXCL4 and CXCL10 can discriminate CMNS from MM $(p<0.0001)$ and CMS $(p<0.0001)$ with an area under the curve $($ AUC $)=1$. These results suggest that CXCL4 and CXCL10 play a prominent role in pathogenesis of CM associated death and may be used as functional or surrogate biomarkers for predicting CM severity.
\end{abstract}

Keywords: CXCL4, CXCL10, PF4, IP-10, malaria, cerebral malaria, Plasmodium falciparum, ROC curve

\section{Introduction}

Cerebral malaria (CM) is a serious complication of Plasmodium falciparum malaria with a wide range of associated neuropathological features [8]. Although

\footnotetext{
* Corresponding author: Jonathan K. Stiles, Morehouse School of Medicine, Department of Microbiology, Biochemistry and Immunology, BMSB Room 349D, 720 Westview Dr. SW, Atlanta, GA 30310, USA. Tel.: +1 404742 1586; Fax: +1 404752 1179; E-mail: jstiles@msm.edu.
}

CM has been studied extensively, many of the pathophysiological mechanisms involved remain unclear and there are limited prognostic tools to determine which infected individual will developed fatal CM [20,39,40, 53,55]. Furthermore, several hypotheses have been proposed to explain the pathophysiology of CM [8, 14] but, none of them by itself explains the pathogenesis. One hypothesis proposes that sequestration of parasitized red blood cells ( $\mathrm{pRBC}$ ) in brain capillaries contiguous to blood-brain barrier (BBB) endothelium results in $\mathrm{BBB}$ dysfunction $[8,10]$. At present, it is not 
clearly understood how sequestered parasites induce the neurological symptoms and mortality associated with CM. However, evidence suggests that parasite sequestration is essential but not sufficient to account for immunopathogenesis of severe form of malaria [58,64] since only a subset of malaria cases, with parasite sequestration succumb to malaria complications [38,72]. In addition, there is evidence to suggest that malaria parasites secrete soluble, apoptotic factor(s) into host blood that could directly mediate neuropathogenesis and BBB damage associated with severe forms of the disease [7,30,75,81]. This observation demonstrates that cytoadhesion of pRBC to host endothelium is not a prerequisite for activation of vascular endothelial cells causing BBB dysfunction [81]. Other studies indicate that the brain lesions are caused by excessive activation of immune cells and the release of pro-inflammatory cytokines which go beyond control [14] and contribute to the pathogenesis of the disease. Our previous studies in West Africa and India revealed for the first time, a striking association between the chemokine CXCL10 (ligand of CXCR3) and CM severity [5,41]. CXCR3 and its ligands have been implicated in several neurological diseases including Toxoplasma gondii [46], West Nile Virus [47,83] and HIV [70]. Recent studies also show that CXCR3 expression is upregulated during malaria infection $[11,36,59,78]$ and that CXCR3-/- mice are relatively more resistant to $P$. berghei ANKA-mediated CM than wild-type controls [11,36,59]. CXCR3 binds four Glutamic acid-Leucine-Arginine (ELR) negative chemokines: platelet factor-4 (CXCL4), monokine induced by interferon- $\gamma$ (CXCL9), interferon- $\gamma$ inducible protein-10 (CXCL10), and interferon-inducible T-cell alpha chemoattractant (CXCL11) all of which exhibit robust angiostatic effects [25].

CXCL4 plays important roles in the control of immunity and inflammation as well as regulation of hematopoiesis and angiogenesis [56]. It is released from alpha-granules of activated platelets during platelet aggregation, and promotes blood coagulation by moderating the effects of heparin-like molecules. Upon activation, platelets interact with endothelial and immune cells promoting further platelet localization and release of inflammatory molecules resulting in vascular inflammation [29,34,78,79]. CXCL4 has also been predicted to play a role in wound repair and inflammation [21]. Among the ligands of CXCR3, CXCL4 is described to be the most powerful angiostatic chemokine [3,63]. Significantly elevated plasma levels of this chemokine have been observed in acute $P$. falciparum infection in human $[22,60]$ and found to mediate murine Experi- mental Cerebral Malaria (ECM) pathogenesis by promoting a pro-inflammatory environment involving increased T cell CXCR3 expression and monocyte activation $[68,69]$.

Expression of CXCL9 is induced by Th1 cytokine, IFN- $\gamma$, and is enhanced in the presence of TNF- $\alpha[23$, 82]. Many cell types (monocytes, neutrophils, endothelial cells, and astrocytes) exhibit IFN- $\gamma$-dependent CXCL9 production $[23,28,33,51]$. CXCL9 has been implicated in pathologies characterized by the accumulation of activated Th1 lymphocytes including autoimmunity, atherosclerosis, psoriasis and allergic contact dermatitis $[27,32,54,66]$.

CXCL11 expression is upregulated in response to IFN- $\gamma$ and enhanced in the presence of IL-1 $\beta$ [15] or TNF- $\alpha$ [74]. There is evidence that CXCL11 may be important in the migration of different subsets of mature thymocytes during thymus lymphopoiesis [62]. It has also been implicated in allergic contact dermatitis [24] and immune-mediated disorders of the central nervous system such as multiple sclerosis [35].

CXCL10 is induced by IFN- $\gamma$ and TNF- $\alpha$ and performs several roles, such as chemoattraction for monocytes and $\mathrm{T}$ cells, promotion of $\mathrm{T}$ cell adhesion to endothelial cells, antitumor activity, inhibition of bone marrow colony formation and angiogenesis [19]. Recent studies measuring 36 serum analytes associated with CM mortality in Ghana and India found that CXCL10 was the only independent molecule that was significantly up-regulated in serum and cerebrospinal fluid (CSF) in Ghanaian children with CM [5,41]. Additionally, CXCL10 and CXCL9 deficient mice were shown to be more resistant to $P$. berghei ANKA infection [11], indicating that both of these CXCR3 ligands mediate development of CM.

Since the CXCR3 receptor shares multiple ligands (CXCL4, CXCL9, CXCL10 and CXCL11), the goal of this study was to determine the key role of CXCR3 ligands associated with fatal CM in order to facilitate their development as biomarker(s) of CM severity. The present study evaluates the predictive value of plasma levels of CXCR3 ligands in determining mortality risk in $\mathrm{CM}$ patients.

\section{Materials and methods}

\subsection{Study sites}

The study was conducted in a malaria-endemic region in Madhya Pradesh, India, which accounts for $23 \%$ of all malaria cases in the state [65]. The study samples were obtained from two sites: Nethaji Subash 
Chandra Bose (NSCB) Hospital (a regional referral hospital) in Jabalpur and Civil Hospital (a primary hospital) in Maihar, Satna District. Both Plasmodium vivax and $P$. falciparum are prevalent in this area, and $P$. falciparum transmission occurs primarily during the monsoon and post-monsoon seasons (July- January). Previous studies revealed that malaria is present in all age groups, with the highest prevalence occurring in children between $8-14$ years of age $[42,67]$.

\subsection{Study subjects}

All subjects were enrolled after informed consent of patients and guardians of patients with unarousable coma were obtained. Informed consent and human subject research guidelines of the National Institutes of Health (NIH, USA), and the ethical committees of the Morehouse School of Medicine (USA), the National Institute of Malaria Research (India), and the Centers for Disease Control and Prevention (CDC) in the United States were followed. Pregnant women and patients with other severe disease such as respiratory distress without $\mathrm{CM}$ and non-CM related coma, were excluded from the study. Data relating to age, sex, and level of parasitemia as well as complications such as seizure and renal failure were obtained from medical records. It was not possible to include data on non-malaria deaths to relate specificity of mortalities to malaria alone. However, recent analyses of CSF and serum in fatal Ghanaian CM cases revealed that the observations were not part of a generalized fatal cascade in human disease [5].

\section{Enrollment criteria}

\subsection{Cerebral malaria}

All CM patients fulfilled the World Health Organization's (WHO) definition of CM [80], and had Glasgow coma score of $\leqslant 8$, a $P$. falciparum parasitemia, and no other clinically evident cause of impaired consciousness [55]. CM survivors (CMS) and non-survivors (CMNS) were separated into two separate groups. All the non-survivors (CMNS) died within three days of admission.

\subsection{Mild malaria}

Mild malaria (MM) patients had fever with $P$. falciparum parasitemia of $<25,000$ parasites/ $\mu$ l of blood (detected microscopically from blood smears) and no evidence of impaired consciousness, seizures, and no past history of mental illness, meningitis, or accidental head injury were included in this group.

\subsection{Healthy control $(H C)$}

Relatives of patients in the hospital and members of the community who did not have malaria or other febrile illness were included after clinical evaluation.

Relevant clinical data and information (such as duration of coma and seizures) were recorded for each patient from physician's records $[41,42]$. Venous blood samples from children $(2-5 \mathrm{ml})$ and adults $(8 \mathrm{ml})$ were collected soon after enrolment into the study at the hospital prior to commencement of anti-malarial treatment. Patients were further classified into HC, MM, CMS, and CMNS groups based on clinical characteristics and treatment outcome. Plasma was separated after centrifugation in Vacutainer Cell Preparation Tubes (CPT) containing Sodium Citrate (Becton Dickinson, USA) for $20 \mathrm{~min}$ at $1500 \mathrm{RCF}$, aliquoted and frozen at $-80^{\circ} \mathrm{C}$ for long-term storage.

\subsection{Patient characteristics}

The plasma samples from $16 \mathrm{HC}, 26 \mathrm{MM}, 26 \mathrm{CMS}$ and 12 CMNS were selected randomly and analyzed. The characteristics of randomly selected subjects are described in Table 1. These subjects were selected from a subset of a prospective study conducted in India [41, 42]. The main complications in CM patients but absent in $\mathrm{HC}$ and MM were seizure (CMS 46\%, CMNS $67 \%$ ) and renal failure (CMS 19\%, CMNS 17\%). Age, sex, and level of parasitemia did not affect prediction of the severity of malaria among the MM, CMS, and CMNS groups (Table 1). Anemia was present in all study groups, but hemoglobin levels were significantly lower in the CMS $(p<0.005)$ and CMNS $(p<0.001)$ patients as compared to HC. Hemoglobin levels were significantly different between MM and CMNS ( $p<$ 0.05 ) but not between MM and CMS groups.

\section{Enzyme-linked immunosorbent assay}

This pilot study utilized plasma samples from a previous study [41], to evaluate CXCL4, CXCL9, CXCL10, and CXCL11 levels by commercially available ELISA kits using human-specific primary and secondary antibodies. IMUCLONE Platelet Factor 4 ELISA was used for CXCL4 assay with minimum detectable limits of $0.5 \mathrm{ng} / \mathrm{mL}$ (American Diagnostica Inc, CT). The CXCL9, CXCL10 and CXCL11 assays were performed by using ELISA kits from R\&D Systems with the following minimum detection 
Table 1

Clinical study characteristics of the study participants in Jabalpur, India. HC - healthy controls, MM - mild malaria, CMS - cerebral malaria survivors, CMNS - cerebral malaria non-survivors. Relevant clinical data and information (such as duration of coma and seizures) were recorded for each patient from physician's records. Venous blood samples from adults $(10 \mathrm{ml})$ were collected soon after enrolment into the study at the hospital from HC, MM, CMS, and CMNS groups prior to commencement of anti-malarial treatment or transfusions. Plasma was separated after centrifugation in Becton-Dickinson cell preparation tubes and aliquoted and frozen at $-80^{\circ} \mathrm{C}$ for long-term storage. SD denote standard deviation

\begin{tabular}{lcccc}
\hline Characteristic & \multicolumn{3}{c}{ Category } \\
\cline { 2 - 5 } & HC & MM & CMS & CMNS \\
\hline Number of subjects & 16 & 26 & 26 & 12 \\
No. of males & 11 & 16 & 14 & 6 \\
No. of females & 5 & 10 & 12 & 6 \\
Mean age \pm 2SD $(\mathrm{yr})$ & $29.6 \pm 5.2$ & $23.3 \pm 10.2$ & $27.9 \pm 12.4$ & $20.0 \pm 16.0$ \\
Mean Glasgow Coma Score & 14 & 14 & $6.7 \pm 2.5$ & $5.2 \pm 2.4$ \\
Seizures & 0 & 0 & 12 & 8 \\
Geomean parasitemia $\pm 2 \mathrm{SD}\left(\mathrm{pRBC} / \mathrm{mm}^{3}\right)$ & 0 & $863.4 \pm 231.1$ & $2256.6 \pm 352.6$ & $1336 \pm 386.2$ \\
Geomean hemoglobin $\pm 2 \mathrm{SD}(\mathrm{g} / \mathrm{dL})$ & $10.6 \pm 0.2$ & $9.1 \pm 1.5$ & $7.8 \pm 2.5$ & $5.7 \pm 2.0$ \\
Renal failure & 0 & 0 & 5 & 2 \\
\hline
\end{tabular}

limits; CXCL9 (1.37-11.31 pg/mL), CXCL10 (0.41$4.46 \mathrm{pg} / \mathrm{mL}$ ), and CXCL11 (3.4-39.7 pg/mL) (R\&D Systems, MN). The results were interpolated from 5parameter-fit standard curves generated using the relevant recombinant human proteins. Samples were tested at a 1:4 dilution.

\section{Statistical analysis}

Statistical analysis was performed with SigmaPlot 2006 (version 10.0) with SigmaStat (version 3.5) integration (Chicago, IL) software for windows. Data were analyzed by Kruskal-Wallis One-Way ANOVA on Ranks using the Dunn's method to compare pairs of group. A value of $P<0.05$ was considered significant. Receiver Operating Characteristic (ROC) curves enables the assessment of the ability of a test to discriminate between individuals with and without disease [2]. ROC curves were used to determine the predictive value of CXCR3 ligands among the different patient groups. The area under the ROC curve (AUC) reflects specificity and sensitivity of a test in discriminating between patients with disease and those without disease [1]. In general the closer the AUC is to 1 the better the overall diagnostic performance of the test, and the closer it is to 0.0 the poorer the test [1].

\section{Results}

\subsection{Plasma levels of CXCR3 ligands in HC, MM, CMS, and CMNS}

Pairwise comparisons were used to determine levels of significance of each ligand in the four groups $(\mathrm{HC}$,
MM, CMS, CMNS). CMNS patients had significantly higher plasma levels of the CXCL4 and CXCL10 compared to HC, MM, and CMS $(p<0.05)$. Mild malaria patients also demonstrated significantly higher levels of CXCL10 compared to CMS (Fig. 1). Furthermore, the levels of CXCL10 in this study were consistent with the levels reported in the previous study [41]. Plasma levels of CXCL11 and CXCL9 revealed no significant changes between MM, CMS, and CMNS (data not shown).

\section{Receiver operating characteristic (ROC) curve analysis of CXCL4 and CXCL10 levels in patients groups}

ROC curve analysis was used to determine the predictive value of the CXCL4 and CXCL10 levels among the groups. CXCL10 independently discriminated between $\mathrm{HC}$ and $\mathrm{MM}$ with an $\mathrm{AUC}=0.82$ and $p<$ 0.001 (Fig. 2 and Table 2). Both ligands (CXCL4 \& CXCL10) could independently discriminate between HC and CMNS $(p<0.0001$ and AUC $=1)$ as shown in Fig. 2 and Table 2. In addition, CXCL4 and CXCL10 could independently discriminate between MM and CMNS, and CMS and CMNS (Fig. 2 and Table 2). CXCL4 could not discriminate statistically between MM and CMS even though CXCL10 has a moderate AUC of 0.78 (Fig. 2 and Table 2).

\section{Discussion}

Cerebral malaria is the most severe neurological complication of malaria infection in humans. However, 

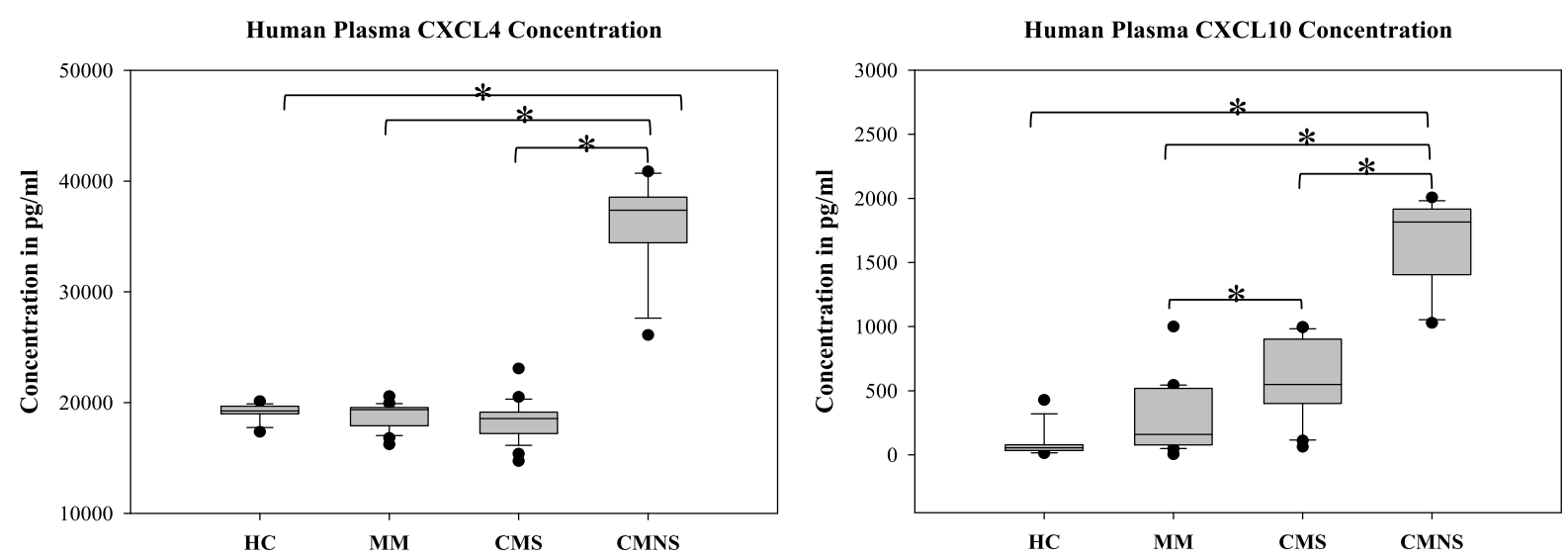

Fig. 1. Plasma levels of CXCL4 and CXCL10 in HC, MM, CMS and CMNs. HC, non-malaria; MM, mild malaria; CMS, cerebral malaria survivor; CMNS, cerebral malaria non-survivor. Box plot represent medians with 25 th and 75 th percentiles, bars for 10th and 90 th percentiles, and points for outliers of CXCL4 and CXCL10 concentrations. Significant differences between CXCS4 and CXCS10 levels in different categories in relation to severity of CM were determined by Kruskal-Wallis One-Way ANOVA on Ranks using the Dunn's method to compare pairs of group. * represent $p<0.05$.

the mechanisms underlying the fatal cerebral complications are still not fully understood. Studies suggest that hyperimmune responses and dysregulation of proand anti-inflammatory cytokines or chemokines result in localized recruitment of immune effector cells and BBB impairment leading to the development of cerebral complication [5]. However, approximately $2 \%$ of malaria infection leads to life-threatening CM [49] and yet there are no laboratory tests that definitively confirm diagnosis of CM [17,73]. The lack of definitive diagnosis of CM due to the non-specific nature of the clinical presentation of the disease may result in misdiagnosis and adverse outcomes $[61,73]$. The discovery of a reliable laboratory test that accurately identifies individuals at risk of developing fatal CM would be a valuable tool. Human studies have revealed tight association between levels of specific chemokines and CM mortality $[4,5,12,41,43-45,67]$. Thus, understanding the alterations in cytokine or chemokine homeostasis in $\mathrm{CM}$ patients will elucidate the underlying pathogenesis and identify potential predictive prognostic biomarkers for CM mortality.

In our previous studies, CXCL10 was the only independent chemokine predictor of fatal CM in Ghanaian children and Indians who died of CM [5,41]. This was the second report from our laboratory to link CXCL10 to fatal CM. This data formed the basis of studies conducted by Campanella et al. [11] who utilized a mouse model of ECM (P. berghei ANKA) to determine that significantly high levels (2 fold) of CXCL10 and CXCL9 expression in brain and spleen of wild-type mice was associated with fatal ECM [11]. CXCR3, a recep- tor for both CXCL10 and CXCL9 mediates a variety of disease states including allograft rejection [35] and lymphocytic choriomeningitis virus [13]. In addition, CXCR3 has been associated with severity of CM [11, 59]. Since CXCR3 is a promiscuous receptor for CXCL10, CXCL9, CXCL11, and CXCL4, their role in CM severity was the focus of this pilot study.

CXCL10 is a chemokine that is induced by IFN- $\gamma$, TNF- $\alpha$, and other factors and has chemotactic activity for activated Th1 lymphocytes [41]. There is a growing appreciation of the role of CXCL10 in both infectious and non-infectious causes of CNS neuronal injury, dementia and inhibition of angiogenesis $[6,26,50,71]$. In this study, CXCL10 levels were remarkably elevated as malaria severity increased with the highest levels present among the CMNS group. These findings are consistent with previous studies conducted in Ghana [5] and India [41] and in the ECM model [11] and confirms the observation linking elevated CXCL10 in CM patients with poor prognosis $[5,41]$. It has been suggested that the release of malaria antigens after schizont rupture activates TNF- $\alpha$ which may activate production of CXCL10 in brain capillaries and astrocytes [18, 37]. High levels of CXCL10 may cause vascular injury resulting in breakdown in the BBB which may lead to accumulation of leukocytes that induce local hyper-inflammation [41]. It has been shown that CXCL10 plays a role in attracting $\mathrm{CD}^{+} \mathrm{T}$ cells and NK cells to brains of infected mice and may be involved in causing death [11]. High levels of CXCL10 have been reported in CSF of viral meningitis patients [50] while elevated CSF level of CXCL10 has been shown 

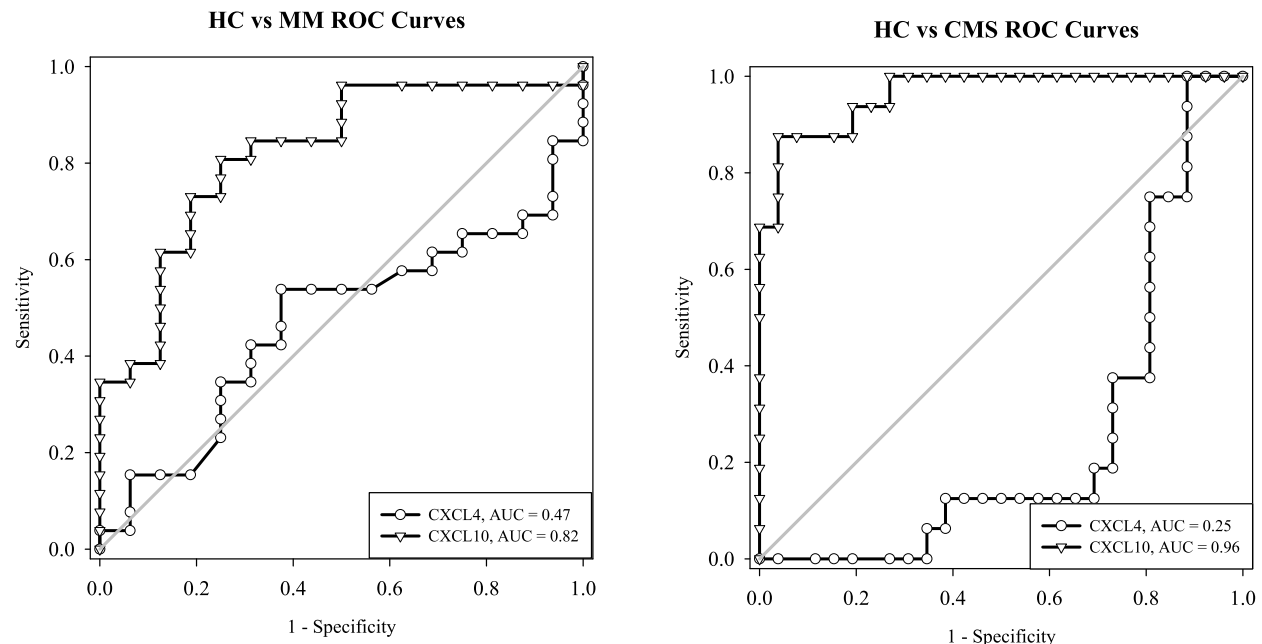

HC vs CMNS ROC Curves

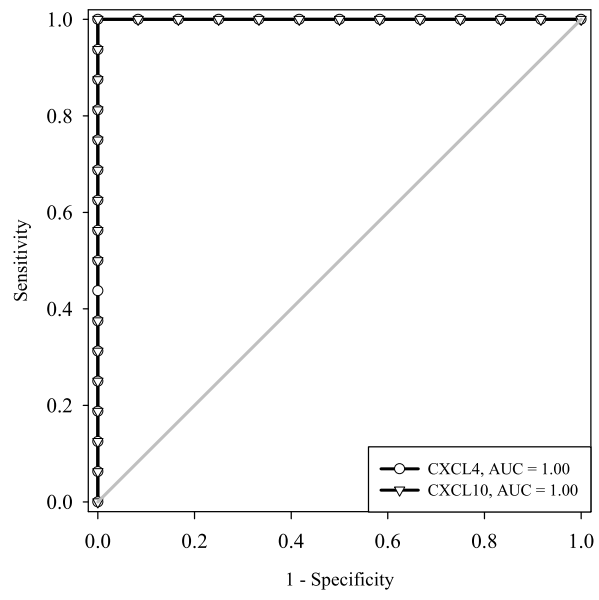

MM vs CMS ROC Curves

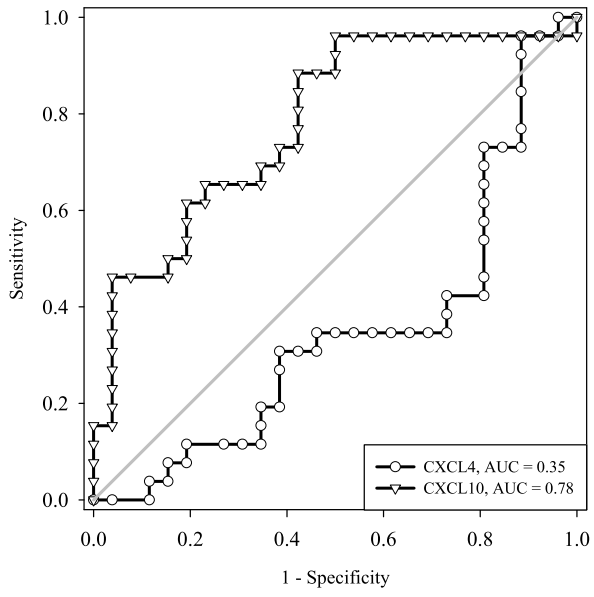

MM vs CMNS ROC Curves

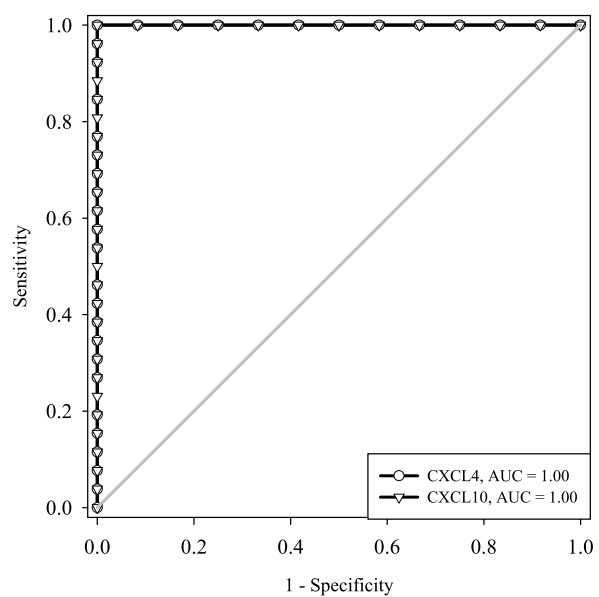

CMS vs CMNS ROC Curves

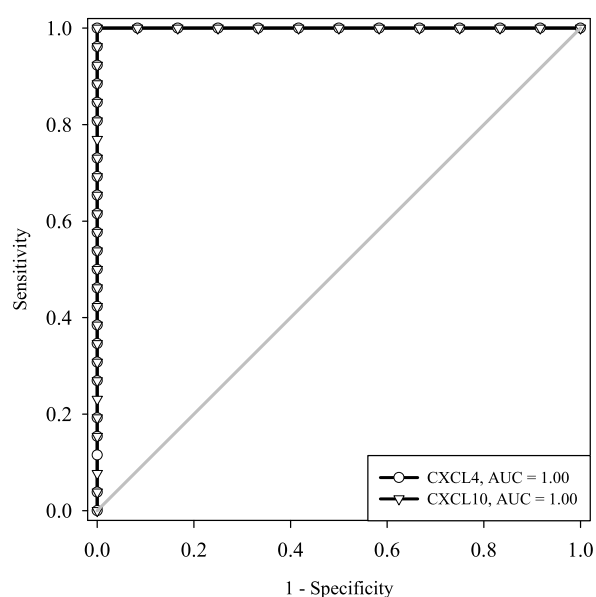

Fig. 2. Receiver Operating characteristic curve analyses of CXCL4 and CXCL10 among the different patient groups. The diagonal line denotes an uninformative test, with an AUC of 0.5 . A test with a perfect discrimination yields an AUC of 1 . Sensitivity refers to the true positive rate and specificity refers to the true negative rate. 
Table 2

Determination of risk of CM severity between different categories by ROC curve analysis. HC - healthy controls, MM - mild malaria, CMS - cerebral malaria survivors, CMNS - cerebral malaria non-survivors. AUC denotes area under the curve and CI denote confidence interval. $P<0.05$ considered statistically significant

\begin{tabular}{lcclcc}
\hline \multirow{2}{*}{ Paired groups } & \multicolumn{2}{c}{ CXCL4 } & & \multicolumn{2}{c}{ CXCL10 } \\
\cline { 2 - 3 } \cline { 5 - 6 } & AUC $(95 \%$ CI $)$ & P-value & & AUC $(95 \%$ CI $)$ & P-value \\
\hline HC vs MM & $0.47(0.29-0.65)$ & 0.75 & & $0.82(0.69-0.95)$ & $<0.001$ \\
HC vs CMS & $0.25(0.10-0.40)$ & 0.007 & & $0.96(0.92-1.00)$ & $<0.0001$ \\
HC vs CMNS & $1.00(1.00-1.00)$ & $<0.0001$ & & $1.00(1.00-1.00)$ & $<0.0001$ \\
MM vs CMS & $0.35(0.19-0.50)$ & 0.05 & & $0.78(0.65-0.91)$ & $<0.001$ \\
MM vs CMNS & $1.00(1.00-1.00)$ & $<0.0001$ & & $1.00(1.00-1.00)$ & $<0.0001$ \\
CMS vs CMNS & $1.00(1.00-1.00)$ & $<0.0001$ & & $1.00(1.00-1.00)$ & $<0.0001$ \\
\hline
\end{tabular}

to be correlated with neuropsychiatric impairment in HIV-associated dementia [48]. Furthermore, mouse studies have shown that HIV-1 virus-encoded protein gp120 directly activates astrocytes to produce CXCL10 using a novel mechanism independent of IFN- $\alpha$ and the STAT-1 pathway of CXCL10 induction [6]. In addition, elevated levels of CXCL10 have been detected in Japanese encephalitis patients [9]. Based on these findings and those from other infectious disease models it is evident that CXCL10 play an important role in disease-induced brain encephalopathy or injury.

Platelets secrete inflammatory mediators when activated [69]. Platelets play important roles in hemostasis and inflammation and potentiate cytotoxicity of pRBC to brain endothelial cells by altering their integrity and increasing apoptosis [69,78]. Mouse studies have shown that platelets have a deleterious role in $\mathrm{CM}$ that may be dependent on the presence of Plasmodium infection through CD36 dependent interactions [34,52, $57,68,76,78]$. In addition, brain lesions of $\mathrm{CM}$ are characterized by sequestration of platelets within brain microvessels, by excessive release of pro-inflammatory cytokines as well as by disruption of the BBB [16, 78]. CXCL4 is released from alpha-granules of activated platelets during platelet aggregation, and promotes blood coagulation by moderating the effects of heparin-like molecules. Recent studies demonstrated that $\mathrm{pRBC}$ activates platelets and stimulate the release of CXCL4 [68]. Platelet accumulation in cerebral microvessels of patients who die from CM reveal a possible role of platelets and CXCL4 in the pathogenesis of CM [34]. Elevated levels of CXCL4 have been associated with acute human malaria [22] and in ECM [68]. CXCL4 stimulates macrophage and TNF- $\alpha$, a key proinflammatory molecule associated with the development of CM $[31,52,78]$. Furthermore, TNF- $\alpha$ increases platelet binding to brain microvasculature during ECM suggesting the importance of platelets in CM patho- genesis $[69,77]$. A recent study indicated that CXCL4 deleteriously drives immune stimulation and monocyte activation in ECM [69]. In this study we demonstrate for the first time that significant elevation of plasma levels of CXCL4 are associated with CM mortality in humans. This supports the evidence that CXCL4 plays a role in CM immunopathogenesis [68,69] and begs for further study in other endemic settings.

Using ROC curves to determine the predictive or discriminating value of CXCL4 and CXCL10, we observed that these ligands accurately predicted or discriminated between CMS and CMNS risk. Moreover, the ROC curve analysis indicated that CXCL10 was the only ligand to accurately discriminate between MM and CMS cases. Surprisingly, CXCL10 was the only ligand that could predict or discriminate between $\mathrm{HC}$ and MM cases using the ROC curve analysis but not in the pairwise comparisons analysis. Recent studies have demonstrated that angiopoietin-1 (ANG-1) and 2 (ANG-2) as well as their ratios (ANG-2:ANG1) are sensitive and specific indicators of severe disease that effectively differentiate between uncomplicated malaria and CM $[17,53]$. However, they could not be used to predict which patients with uncomplicated disease progress to fatal CM [53]. However, in this study, CXCL10 accurately discriminated between MM and CMS. In addition, CXCL10 and CXCL4 accurately predicted or discriminated between CMS and CMNS risk suggesting that these two biomarkers could predict patients who will develop fatal CM.

High systemic levels of CXCL4 and CXCL10, with their strong angiostatic properties in CM patients may further inhibit angiogenesis and regeneration of damaged blood capillaries thereby exacerbating BBB dysfunction and severity of the disease. The findings of elevated CXCL4 and CXCL10 in CM provide new insights on the contribution of Plasmodium induced host factors in mortality associated with the disease. Col- 
lectively, these results suggest a prominent role for $\mathrm{CX}$ CL4 and CXCL10 in the pathogenesis of fatal CM. CXCL10 and CXCL4 will be ideal biomarkers for fatal CM because they are easily measured in serum or plasma, have high sensitivity and specificity in individuals with either CM or at risk of developing fatal CM. furthermore, they are involved in the underlying pathogenesis of CM and may represent potential therapeutic targets for intervention [5,11,41,53,68,69].

In conclusion, this pilot study has revealed new associations between the CXCR3 ligands, CXCL4 and CXCL10, and CM mortality. This study demonstrates that elevated levels of CXCL10 and CXCL4 may be used as functional or surrogate biomarkers in association with positive malaria status for predicting severity associated with CM. However, before CXCL10 and CXCL4 may be developed into diagnostic panels for malaria and clinical practice, additional prospective studies are needed to confirm and validate their usefulness in different populations. Optimal cut-offs need to be determined using standardized methods of sample collection, processing and laboratory protocols for biomarker testing [17]. Furthermore, the study indicates that platelet activation plays a role in driving the pathogenesis of CM. Identifying the functional role of platelet and platelet derived factors in CM may lead to the development of therapeutic strategies to reduce severity of malaria.

\section{Acknowledgements}

We thank the former Director General, Indian Council of Medical Research (ICMR), Dr. N.K. Ganguly and other ICMR Headquarters staff for their support. The authors acknowledge the patients, CM victims and their guardians for providing permission to use their samples. The authors are grateful to the staff of Nethaji Subash Chandra Bose (NSCB) Hospital (Jabalpur) and Civil Hospital (Maihar) and the support of the Center for Disease Diagnosis and Research (CDDR) in Ghana.

\section{Financial support}

This investigation received financial support from WHO/UNDP/TDR Collaborative Research Grant (A00 524) and National Institutes of Health grant numbers NIH-FIC (R21TW006804-01), NIH-RCMI (RR03034) and NIH-NIGM-MBRS (SO6GM08248).

\section{References}

[1] A.K. Akobeng, Understanding diagnostic tests 3: Receiver operating characteristic curves, Acta Paediatr 96 (2007), 644647.

[2] D.G. Altman and J.M. Bland, Diagnostic tests 3: receiver operating characteristic plots, BMJ 309 (1994), 188.

[3] D.A. Arenberg, S.L. Kunkel, P.J. Polverini, S.B. Morris, M.D. Burdick, M.C. Glass, D.T. Taub, M.D. Iannettoni, R.I. Whyte and R.M. Strieter, Interferon-gamma-inducible protein 10 (IP$10)$ is an angiostatic factor that inhibits human non-small cell lung cancer (NSCLC) tumorigenesis and spontaneous metastases, J Exp Med 184 (1996), 981-992.

[4] H. Armah, A.K. Dodoo, E.K. Wiredu, J.K. Stiles, A.A. Adjei, R.K. Gyasi and Y. Tettey, High-level cerebellar expression of cytokines and adhesion molecules in fatal, paediatric, cerebral malaria, Ann Trop Med Parasitol 99 (2005), 629-647.

[5] H.B. Armah, N.O. Wilson, B.Y. Sarfo, M.D. Powell, V.C. Bond, W. Anderson, A.A. Adjei, R.K. Gyasi, Y. Tettey, E.K. Wiredu, J.E. Tongren, V. Udhayakumar and J.K. Stiles, Cerebrospinal fluid and serum biomarkers of cerebral malaria mortality in Ghanaian children, Malar J 6 (2007), 147.

[6] V.C. Asensio, J. Maier, R. Milner, K. Boztug, C. Kincaid, M. Moulard, C. Phillipson, K. Lindsley, T. Krucker, H.S. Fox and I.L. Campbell, Interferon-independent, human immunodeficiency virus type 1 gp120-mediated induction of CXCL10/IP10 gene expression by astrocytes in vivo and in vitro, $J$ Virol 75 (2001), 7067-7077.

[7] A.T. Balde, G. Aribot, A. Tall, A. Spiegel and C. Roussilhon, Apoptosis modulation in mononuclear cells recovered from individuals exposed to Plasmodium falciparum infection, Parasite Immunol 22 (2000), 307-318.

[8] A.R. Berendt, G.D. Tumer and C.I. Newbold, Cerebral malaria: the sequestration hypothesis, Parasitol Today 10 (1994), $412-414$.

[9] S. Bhowmick, R. Duseja, S. Das, M.B. Appaiahgiri, S. Vrati and A. Basu, Induction of IP-10 (CXCL10) in astrocytes following Japanese encephalitis, Neurosci Lett 414 (2007), 4550.

[10] H. Brown, T.T. Hien, N. Day, N.T. Mai, L.V. Chuong, T.T. Chau, P.P. Loc, N.H. Phu, D. Bethell, J. Farrar, K. Gatter, N. White and G. Turner, Evidence of blood-brain barrier dysfunction in human cerebral malaria, Neuropathol Appl Neurobiol 25 (1999), 331-340.

[11] G.S. Campanella, A.M. Tager, J.K. El Khoury, S.Y. Thomas, T.A. Abrazinski, L.A. Manice, R.A. Colvin and A.D. Luster, Chemokine receptor CXCR3 and its ligands CXCL9 and CXCL10 are required for the development of murine cerebral malaria, Proc Natl Acad Sci U S A 105 (2008), 4814-4819.

[12] C. Casals-Pascual, R. Idro, N. Gicheru, S. Gwer, B. Kitsao, E. Gitau, R. Mwakesi, D.J. Roberts and C.R. Newton, High levels of erythropoietin are associated with protection against neurological sequelae in African children with cerebral malaria, Proc Natl Acad Sci U S A 105 (2008), 2634-2639.

[13] J.E. Christensen, A. Nansen, T. Moos, B. Lu, C. Gerard, J.P. Christensen and A.R. Thomsen, Efficient T-cell surveillance of the CNS requires expression of the CXC chemokine receptor 3, J Neurosci 24 (2004), 4849-4858.

[14] I.A. Clark and K.A. Rockett, The cytokine theory of human cerebral malaria, Parasitol Today 10 (1994), 410-412.

[15] K.E. Cole, C.A. Strick, T.J. Paradis, K.T. Ogborne, M. Loetscher, R.P. Gladue, W. Lin, J.G. Boyd, B. Moser, D.E. Wood, B.G. Sahagan and K. Neote, Interferon-inducible T cell alpha chemoattractant (I-TAC): a novel non-ELR CXC 
chemokine with potent activity on activated $\mathrm{T}$ cells through selective high affinity binding to CXCR3, J Exp Med 187 (1998), 2009-2021.

[16] V. Combes, N. Coltel, D. Faille, S.C. Wassmer and G.E. Grau, Cerebral malaria: role of microparticles and platelets in alterations of the blood-brain barrier, Int J Parasitol 36 (2006), 541-546.

[17] A.L. Conroy, E.I. Lafferty, F.E. Lovegrove, S. Krudsood, N. Tangpukdee, W.C. Liles and K.C. Kain, Whole blood angiopoietin-1 and -2 levels discriminate cerebral and severe (non-cerebral) malaria from uncomplicated malaria, Malar $J$ 8 (2009), 295

[18] J. Croitoru-Lamoury, G.J. Guillemin, F.D. Boussin, B. Mognetti, L.I. Gigout, A. Cheret, B. Vaslin, G.R. Le, B.J. Brew and D. Dormont, Expression of chemokines and their receptors in human and simian astrocytes: evidence for a central role of TNF alpha and IFN gamma in CXCR4 and CCR5 modulation, Glia 41 (2003), 354-370.

[19] J.H. Dufour, M. Dziejman, M.T. Liu, J.H. Leung, T.E. Lane and A.D. Luster, IFN-gamma-inducible protein 10 (IP-10; CXCL10)-deficient mice reveal a role for IP-10 in effector T cell generation and trafficking, J Immunol 168 (2002), 31953204.

[20] A. Dzeing-Ella, P.C. Nze Obiang, R. Tchoua, T. Planche, B. Mboza, M. Mbounja, U. Muller-Roemer, J. Jarvis, E. Kendjo, E. Ngou-Milama, P.G. Kremsner, S. Krishna and M. Kombila, Severe falciparum malaria in Gabonese children: clinical and laboratory features, Malar J 4 (2005), 1.

[21] R. Eisman, S. Surrey, B. Ramachandran, E. Schwartz and M. Poncz, Structural and functional comparison of the genes for human platelet factor 4 and PF4alt, Blood 76 (1990), 336-344.

[22] E.M. Essien and M.I. Ebhota, Platelet secretory activities in acute malaria (Plasmodium falciparum) infection, Acta Haematol 70 (1983), 183-188.

[23] J.M. Farber, A macrophage mRNA selectively induced by gamma-interferon encodes a member of the platelet factor 4 family of cytokines, Proc Natl Acad Sci U S A 87 (1990), 5238-5242.

[24] J. Flier, D.M. Boorsma, D.P. Bruynzeel, P.J. Van Beek, T.J. Stoof, R.J. Scheper, R. Willemze and C.P. Tensen, The CXCR3 activating chemokines IP-10, Mig, and IP-9 are expressed in allergic but not in irritant patch test reactions, J Invest Dermatol 113 (1999), 574-578.

[25] N.G. Frangogiannis, Chemokines in ischemia and reperfusion, Thromb Haemost 97 (2007), 738-747.

[26] D. Galimberti, N. Schoonenboom, P. Scheltens, C. Fenoglio, F. Bouwman, E. Venturelli, I. Guidi, M.A. Blankenstein, N. Bresolin and E. Scarpini, Intrathecal chemokine synthesis in mild cognitive impairment and Alzheimer disease, Arch Neurol 63 (2006), 538-543.

[27] M.A. Garcia-Lopez, D. Sancho, F. Sanchez-Madrid and M. Marazuela, Thyrocytes from autoimmune thyroid disorders produce the chemokines IP-10 and Mig and attract CXCR3+ lymphocytes, J Clin Endocrinol Metab 86 (2001), 5008-5016.

[28] S. Gasperini, M. Marchi, F. Calzetti, C. Laudanna, L. Vicentini, H. Olsen, M. Murphy, F. Liao, J. Farber and M.A. Cassatella, Gene expression and production of the monokine induced by IFN-gamma (MIG), IFN-inducible T cell alpha chemoattractant (I-TAC), and IFN-gamma-inducible protein10 (IP-10) chemokines by human neutrophils, J Immunol 162 (1999), 4928-4937.

[29] M. Gawaz, H. Langer and A.E. May, Platelets in inflammation and atherogenesis, J Clin Invest 115 (2005), 3378-3384.
[30] D. Ghigo, R. Todde, H. Ginsburg, C. Costamagna, P. Gautret, F. Bussolino, D. Ulliers, G. Giribaldi, E. Deharo, G. Gabrielli, G. Pescarmona and A. Bosia, Erythrocyte stages of Plasmodium falciparum exhibit a high nitric oxide synthase (NOS) activity and release an NOS-inducing soluble factor, J Exp Med 182 (1995), 677-688.

[31] F. Gimenez, L.S. Barraud de, C. Fernandez, P. Pino and D. Mazier, Tumor necrosis factor alpha in the pathogenesis of cerebral malaria, Cell Mol Life Sci 60 (2003), 1623-1635.

[32] M. Goebeler, A. Toksoy, U. Spandau, E. Engelhardt, E.B. Brocker and R. Gillitzer, The C-X-C chemokine Mig is highly expressed in the papillae of psoriatic lesions, J Pathol 184 (1998), 89-95.

[33] M. Goebeler, A. Trautmann, A. Voss, E.V. Brocker, A. Toksoy and R. Gillitzer, Differential and sequential expression of multiple chemokines during elicitation of allergic contact hypersensitivity, Am J Pathol 158 (2001), 431-440.

[34] G.E. Grau, C.D. Mackenzie, R.A. Carr, M. Redard, G. Pizzolato, C. Allasia, C. Cataldo, T.E. Taylor and M.E. Molyneux, Platelet accumulation in brain microvessels in fatal pediatric cerebral malaria, J Infect Dis 187 (2003), 461-466.

[35] W.W. Hancock, B. Lu, W. Gao, V. Csizmadia, K. Faia, J.A. King, S.T. Smiley, M. Ling, N.P. Gerard and C. Gerard, Requirement of the chemokine receptor CXCR3 for acute allograft rejection, J Exp Med 192 (2000), 1515-1520.

[36] D.S. Hansen, N.J. Bernard, C.Q. Nie and L. Schofield, NK cells stimulate recruitment of CXCR3 $+\mathrm{T}$ cells to the brain during Plasmodium berghei-mediated cerebral malaria, J Immunol 178 (2007), 5779-5788.

[37] P.S. Hanum, M. Hayano and S. Kojima, Cytokine and chemokine responses in a cerebral malaria-susceptible or resistant strain of mice to Plasmodium berghei ANKA infection: early chemokine expression in the brain, Int Immunol $\mathbf{1 5}$ (2003), 633-640.

[38] R. Idro, N.E. Jenkins and C.R. Newton, Pathogenesis, clinical features, and neurological outcome of cerebral malaria, Lancet Neurol 4 (2005), 827-840.

[39] R. Idro, C. Karamagi and J. Tumwine, Immediate outcome and prognostic factors for cerebral malaria among children admitted to Mulago Hospital, Uganda, Ann Trop Paediatr 24 (2004), 17-24.

[40] S. Jaffar, M.B. Van Hensbroek, A. Palmer, G. Schneider and B. Greenwood, Predictors of a fatal outcome following childhood cerebral malaria, Am J Trop Med Hyg 57 (1997), 20-24.

[41] V. Jain, H.B. Armah, J.E. Tongren, R.M. Ned, N.O. Wilson, S. Crawford, P.K. Joel, M.P. Singh, A.C. Nagpal, A.P. Dash, V. Udhayakumar, N. Singh and J.K. Stiles, Plasma IP-10, apoptotic and angiogenic factors associated with fatal cerebral malaria in India, Malar J 7 (2008), 83.

[42] V. Jain, A.C. Nagpal, P.K. Joel, M. Shukla, M.P. Singh, R.B. Gupta, A.P. Dash, S.K. Mishra, V. Udhayakumar, J.K. Stiles and N. Singh, Burden of cerebral malaria in central India (2004-2007), Am J Trop Med Hyg 79 (2008), 636-642.

[43] C.C. John, R. Opika-Opoka, J. Byarugaba, R. Idro and M.J. Boivin, Low levels of RANTES are associated with mortality in children with cerebral malaria, J Infect Dis 194 (2006), 837-845.

[44] C.C. John, A. Panoskaltsis-Mortari, R.O. Opoka, G.S. Park, P.J. Orchard, A.M. Jurek, R. Idro, J. Byarugaba and M.J. Boivin, Cerebrospinal fluid cytokine levels and cognitive impairment in cerebral malaria, Am J Trop Med Hyg 78 (2008), 198-205.

[45] C.C. John, G.S. Park, N. Sam-Agudu, R.O. Opoka and M.J. Boivin, Elevated serum levels of IL-1ra in children with Plas- 
modium falciparum malaria are associated with increased severity of disease, Cytokine 41 (2008), 204-208.

[46] I.A. Khan, J.A. MacLean, F.S. Lee, L. Casciotti, E. DeHaan, J.D. Schwartzman and A.D. Luster, IP-10 is critical for effector $\mathrm{T}$ cell trafficking and host survival in Toxoplasma gondii infection, Immunity 12 (2000), 483-494.

[47] R.S. Klein, E. Lin, B. Zhang, A.D. Luster, J. Tollett, M.A. Samuel, M. Engle, and M.S. Diamond, Neuronal CXCL10 directs CD8+ T-cell recruitment and control of West Nile virus encephalitis, J Virol 79 (2005), 11457-11466.

[48] S.A. Kolb, B. Sporer, F. Lahrtz, U. Koedel, H.W. Pfister and $\mathrm{A}$. Fontana, Identification of a $\mathrm{T}$ cell chemotactic factor in the cerebrospinal fluid of HIV-1-infected individuals as interferon-gamma inducible protein 10, J Neuroimmunol 93 (1999), 172-181.

[49] D.P. Kwiatkowski, How malaria has affected the human genome and what human genetics can teach us about malaria, Am J Hum Genet 77 (2005), 171-192.

[50] F. Lahrtz, L. Piali, D. Nadal, H.W. Pfister, K.S. Spanaus, M. Baggiolini and A. Fontana, Chemotactic activity on mononuclear cells in the cerebrospinal fluid of patients with viral meningitis is mediated by interferon-gamma inducible protein-10 and monocyte chemotactic protein-1, Eur J Immunol 27 (1997), 2484-2489.

[51] F. Liao, R.L. Rabin, J.R. Yannelli, L.G. Koniaris, P. Vanguri and J.M. Farber, Human Mig chemokine: biochemical and functional characterization, J Exp Med 182 (1995), 13011314.

[52] J. Lou, Y.R. Donati, P. Juillard, C. Giroud, C. Vesin, N. Mili and G.E. Grau, Platelets play an important role in TNFinduced microvascular endothelial cell pathology, Am J Pathol 151 (1997), 1397-1405.

[53] F.E. Lovegrove, N. Tangpukdee, R.O. Opoka, E.I. Lafferty, N. Rajwans, M. Hawkes, S. Krudsood, S. Looareesuwan, C.C. John, W.C. Liles and K.C. Kain, Serum angiopoietin-1 and -2 levels discriminate cerebral malaria from uncomplicated malaria and predict clinical outcome in African children, PLoS One 4 (2009), e4912.

[54] F. Mach, A. Sauty, A.S. Iarossi, G.K. Sukhova, K. Neote, P. Libby and A.D. Luster, Differential expression of three T lymphocyte-activating CXC chemokines by human atheromaassociated cells, J Clin Invest 104 (1999), 1041-1050.

[55] K. Marsh, D. Forster, C. Waruiru, I. Mwangi, M. Winstanley, V. Marsh, C. Newton, P. Winstanley, P. Warn and N. Peshu, Indicators of life-threatening malaria in African children, $N$ Engl J Med 332 (1995), 1399-1404.

[56] A.M. Maurer, B. Zhou and Z.C. Han, Roles of platelet factor 4 in hematopoiesis and angiogenesis, Growth Factors 24 (2006), 242-252.

[57] B.J. McMorran, V.M. Marshall, G.C. de, K.E. Drysdale, M. Shabbar, G.K. Smyth, J.E. Corbin, W.S. Alexander and S.J. Foote, Platelets kill intraerythrocytic malarial parasites and mediate survival to infection, Science 323 (2009), 797-800.

[58] D.A. Milner, Jr., C.P. Dzamalala, N.G. Liomba, M.E. Molyneux and T.E. Taylor, Sampling of supraorbital brain tissue after death: improving on the clinical diagnosis of cerebral malaria, J Infect Dis 191 (2005), 805-808.

[59] J. Miu, A.J. Mitchell, M. Muller, S.L. Carter, P.M. Manders, J.A. McQuillan, B.M. Saunders, H.J. Ball, B. Lu, I.L. Campbell and N.H. Hunt, Chemokine gene expression during fatal murine cerebral malaria and protection due to CXCR3 deficiency, J Immunol 180 (2008), 1217-1230.

[60] B. Polack, F. Peyron, Z. Sheick, I.L. Kolodie and P. mbroiseThomas, [Erythrocytes infected by Plasmodium falciparum activate human platelets], C R Acad Sci III 310 (1990), 577582.

61] H. Reyburn, R. Mbatia, C. Drakeley, I. Carneiro, E. Mwakasungula, O. Mwerinde, K. Saganda, J. Shao, A. Kitua, R. Olomi, B.M. Greenwood and C.J. Whitty, Overdiagnosis of malaria in patients with severe febrile illness in Tanzania: a prospective study, BMJ 329 (2004), 1212.

[62] P. Romagnani, F. Annunziato, E. Lazzeri, L. Cosmi, C. Beltrame, L. Lasagni, G. Galli, M. Francalanci, R. Manetti, F. Marra, V. Vanini, E. Maggi and S. Romagnani, Interferoninducible protein 10 , monokine induced by interferon gamma, and interferon-inducible T-cell alpha chemoattractant are produced by thymic epithelial cells and attract T-cell receptor (TCR) alphabeta+ CD8+ single-positive T cells, TCRgammadelta+ T cells, and natural killer-type cells in human thymus, Blood 97 (2001), 601-607.

[63] M.M. Rosenkilde and T.W. Schwartz, The chemokine system a major regulator of angiogenesis in health and disease, APMIS 112 (2004), 481-495.

[64] K.B. Seydel, D.A. Milner, Jr., S.B. Kamiza, M.E. Molyneux and T.E. Taylor, The distribution and intensity of parasite sequestration in comatose Malawian children, J Infect Dis 194 (2006), 208-205.

[65] V.P. Sharma, Re-emergence of malaria in India, Indian J Med Res 103 (1996), 26-45.

[66] J.E. Simpson, J. Newcombe, M.L. Cuzner and M.N. Woodroofe, Expression of the interferon-gamma-inducible chemokines IP-10 and Mig and their receptor, CXCR3, in multiple sclerosis lesions, Neuropathol Appl Neurobiol 26 (2000), 133-142.

[67] S. Singh, N. Singh and R. Handa, Tumor necrosis factor-alpha in patients with malaria, Indian J Malariol 37 (2000), 27-33.

[68] K. Srivastava, I.A. Cockburn, A. Swaim, L.E. Thompson, A. Tripathi, C.A. Fletcher, E.M. Shirk, H. Sun, M.A. Kowalska, K. Fox-Talbot, D. Sullivan, F. Zavala and C.N. Morrell, Platelet factor 4 mediates inflammation in experimental cerebral malaria, Cell Host. Microbe 4 (2008), 179-187.

[69] K. Srivastava, D.J. Field, A. Aggrey, M. Yamakuchi and C.N. Morrell, Platelet factor 4 regulation of monocyte KLF4 in experimental cerebral malaria, PLoS One 5 (2010), e10413.

[70] Y. Sui, R. Potula, N. Dhillon, D. Pinson, S. Li, A. Nath, C. Anderson, J. Turchan, D. Kolson, O. Narayan and S. Buch, Neuronal apoptosis is mediated by CXCL10 overexpression in simian human immunodeficiency virus encephalitis, Am J Pathol 164 (2004), 1557-1566.

[71] Y. Sui, R. Potula, D. Pinson, I. Adany, Z. Li, J. Day, E. Buch, J. Segebrecht, F. Villinger, Z. Liu, M. Huang, O. Narayan and S. Buch, Microarray analysis of cytokine and chemokine genes in the brains of macaques with SHIV-encephalitis, $J$ Med Primatol 32 (2003), 229-239.

[72] Z. Taoufiq, F. Gay, J. Balvanyos, L. Ciceron, M. Tefit, P. Lechat and D. Mazier, Rho kinase inhibition in severe malaria: thwarting parasite-induced collateral damage to endothelia, $J$ Infect Dis 197 (2008), 1062-1073.

[73] T.E. Taylor, W.J. Fu, R.A. Carr, R.O. Whitten, J.S. Mueller, N.G. Fosiko, S. Lewallen, N.G. Liomba and M.E. Molyneux, Differentiating the pathologies of cerebral malaria by postmortem parasite counts, Nat Med 10 (2004), 143-145.

[74] C.P. Tensen, J. Flier, E.M. Van Der Raaij-Helmer, S. SampatSardjoepersad, R.C. Van Der Schors, R. Leurs, R.J. Scheper, D.M. Boorsma and R. Willemze, Human IP-9: A keratinocytederived high affinity CXC-chemokine ligand for the IP-10/Mig receptor (CXCR3), J Invest Dermatol 112 (1999), 716-722. 
[75] A. Toure-Balde, J.L. Sarthou, G. Aribot, P. Michel, J.F. Trape, C. Rogier and C. Roussilhon, Plasmodium falciparum induces apoptosis in human mononuclear cells, Infect Immun 64 (1996), 744-750

[76] H.C. van der Heyde, I. Gramaglia, G. Sun and C. Woods, Platelet depletion by anti-CD41 (alphaIIb) mAb injection early but not late in the course of disease protects against Plasmodium berghei pathogenesis by altering the levels of pathogenic cytokines, Blood 105 (2005), 1956-1963.

[77] M.C. von Zur, N.R. Sibson, K. Peter, S.J. Campbell, P. Wilainam, G.E. Grau, C. Bode, R.P. Choudhury and D.C. Anthony, A contrast agent recognizing activated platelets reveals murine cerebral malaria pathology undetectable by conventional MRI, J Clin Invest 118 (2008), 1198-1207.

[78] S.C. Wassmer, V. Combes, F.J. Candal, I. Juhan-Vague and G.E. Grau, Platelets potentiate brain endothelial alterations induced by Plasmodium falciparum, Infect Immun 74 (2006), 645-653.

[79] S.C. Wassmer, V. Combes and G.E. Grau, Pathophysiology of cerebral malaria: role of host cells in the modulation of cytoadhesion, Ann N Y Acad Sci 992 (2003), 30-38.

80] WHO, Severe falciparum malaria. World Health Organization, Communicable Diseases Cluster, Trans $R$ Soc Trop Med Hyg 94(Suppl 1) (2000), S1-90.

[81] N.O. Wilson, M.B. Huang, W. Anderson, V. Bond, M. Powell, W.E. Thompson, H.B. Armah, A.A. Adjei, R. Gyasi, Y. Tettey and J.K. Stiles, Soluble factors from Plasmodium falciparuminfected erythrocytes induce apoptosis in human brain vascular endothelial and neuroglia cells, Mol Biochem Parasitol 162 (2008), 172-176.

[82] P. Wong, C.W. Severns, N.B. Guyer and T.M. Wright, A unique palindromic element mediates gamma interferon induction of mig gene expression, Mol Cell Biol 14 (1994), 914-922.

[83] B. Zhang, Y.K. Chan, B. Lu, M.S. Diamond and R.S. Klein, CXCR3 mediates region-specific antiviral $\mathrm{T}$ cell trafficking within the central nervous system during West Nile virus encephalitis, J Immunol 180 (2008), 2641-2649. 


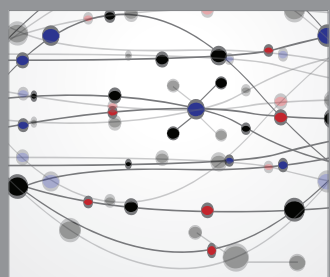

The Scientific World Journal
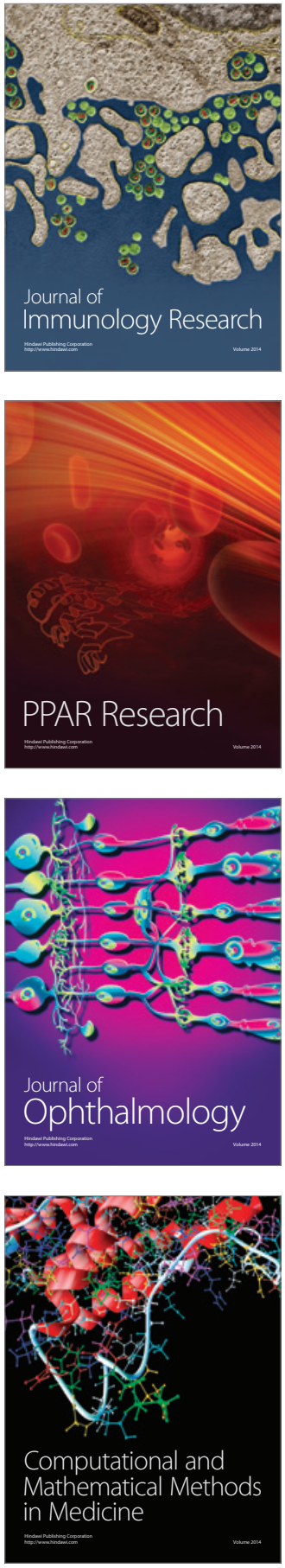

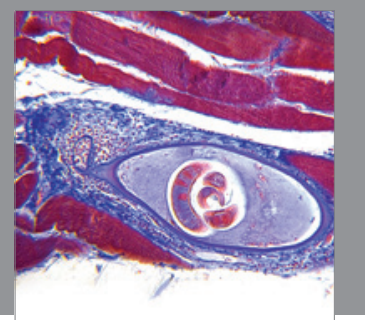

Gastroenterology

Research and Practice
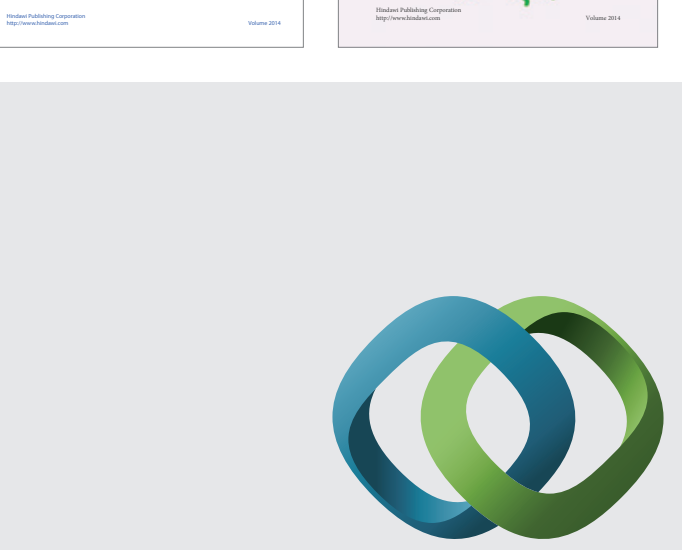

\section{Hindawi}

Submit your manuscripts at

http://www.hindawi.com
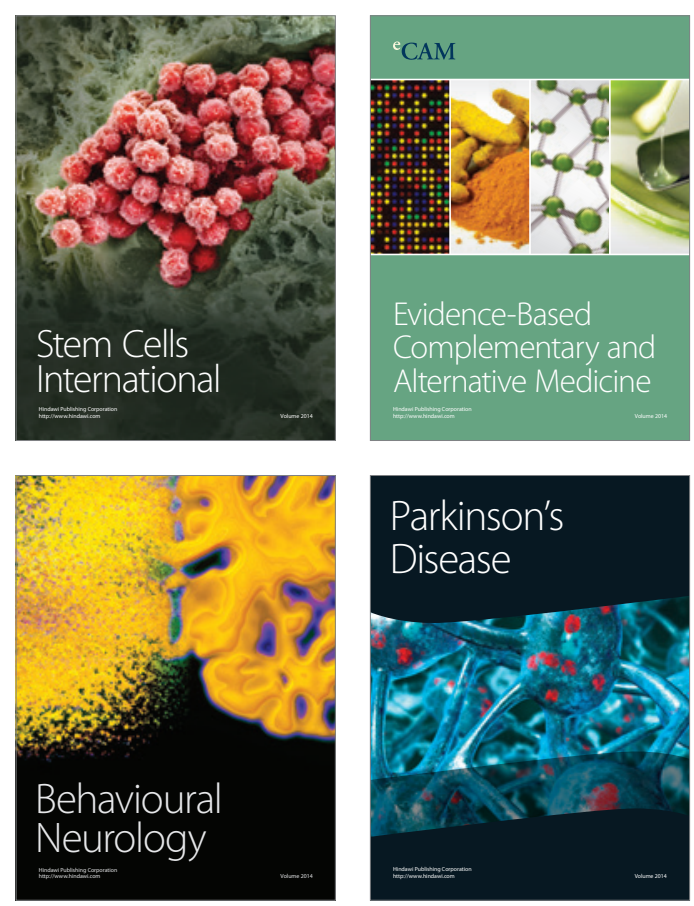

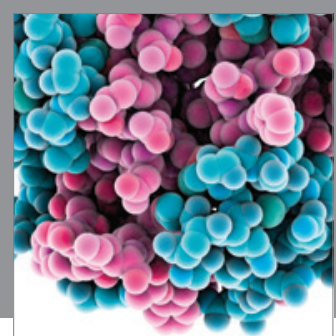

Journal of
Diabetes Research

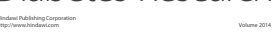

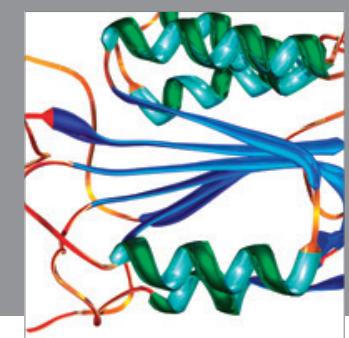

Disease Markers
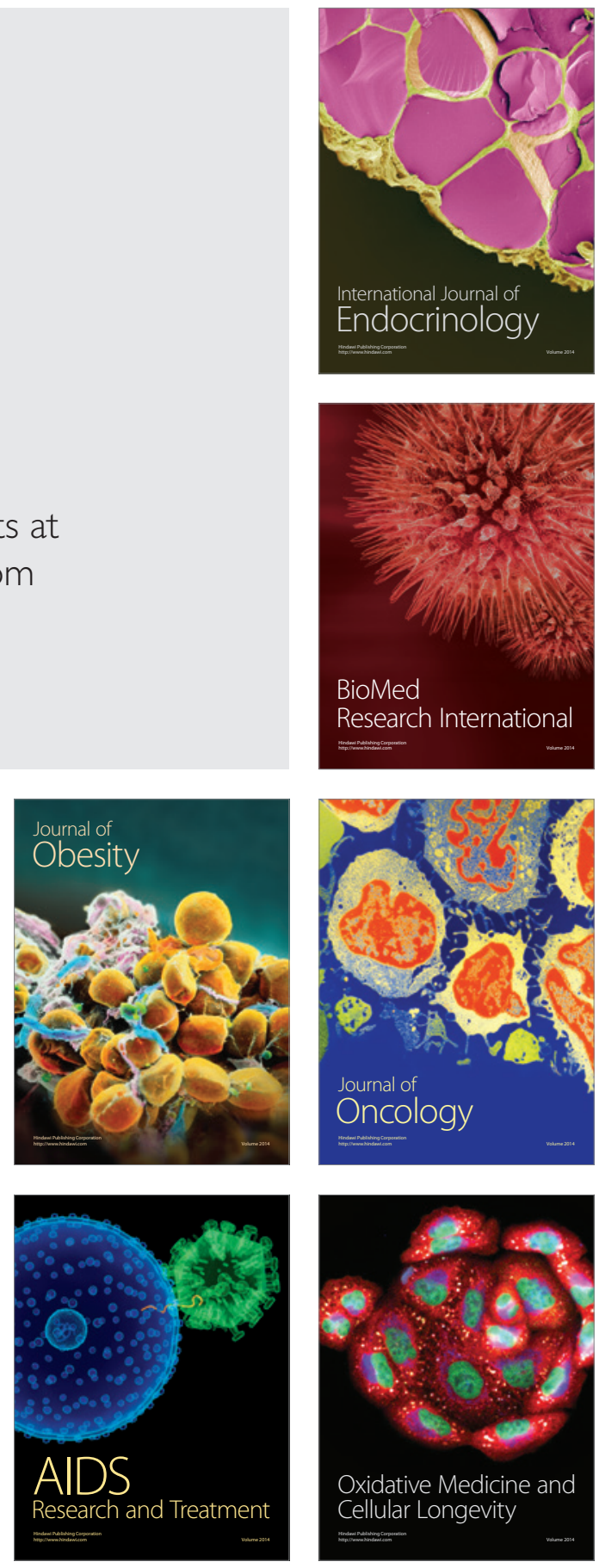